\title{
Sobre la PREsencia de MitTENothamnium REDUNCUM (HypnaCeAe) EN LA ARgentina
}

\author{
MARIA T. COLOTTI', GUILLERMO M. SUAREZ ${ }^{1,2}$ y ALFONS SCHÄFER-VERWIMP³
}

\begin{abstract}
Summary: On the presence of Mittenothamnium reduncum (Hypnaceae) in Argentina. Mittenothamnium reduncum is recorded for the first time for the bryological flora of Argentina. A brief description, illustration, and discussion of the species are provided. Morphological differences between this taxon and M. reptans, the most frequent species in the area, are presented.
\end{abstract}

Key words: New records, National Park Campo de Los Alisos, Taxonomy.

Resumen: Mittenothamnium reduncum, un nuevo registro para la flora briológica de Argentina, se describe, ilustra y comenta brevemente. Se presentan las diferencias morfológicas entre este taxón y $M$. reptans, la especie más frecuente en el área.

Palabras clave: Nuevo registro, Parque Nacional Campo de Los Alisos, Taxonomía.

\section{INTRODUCCIÓN}

El Parque Nacional Campo de los Alisos está situado en el sudoeste de la provincia de Tucumán, en el departamento Chicligasta $\left(27^{\circ} 13^{\prime} \mathrm{S} ; 65^{\circ}\right.$ $\left.58^{\prime} \mathrm{O}\right)$. Toda el área se ubica en la ladera oriental de los Nevados del Aconquija y su superficie de aproximadamente 10.000 hectáreas, se extiende en sentido este-oeste a lo largo de una franja angosta que desciende desde los $5.200 \mathrm{~m}$ en su punto más alto hasta los $680 \mathrm{~m}$ en su menor altura. En el área se mezclan eco regiones de las yungas y altoandinas, destacándose importantes ambientes de transición surcados por varios cursos de agua que en su mayoría tienen rumbo oeste- sudeste.

De la revisión de los ejemplares coleccionados en este parque surgieron importantes novedades para el país, entre ellos Rhamphidium dicranoides

\footnotetext{
1 Facultad de Ciencias Naturales e IML, Universidad Nacional de Tucumán, Miguel Lillo 205, (4000) San Miguel de Tucumán, Tucumán, Argentina; t_colotti@ hotmail.com (autor corresponsal).

2 Unidad Ejecutora Lillo, (CONICET- Fundación Miguel Lillo), Miguel Lillo 251, (4000) San Miguel de Tucumán, Tucumán, Argentina.

3 Mittlere Letten 11, 88634 Herdwangen-Schönach, Germany
}

(Müll. Hal) Paris (Colotti \& Suárez, 2017). Recientemente se ha encontrado un espécimen de Mittenothamnium Henn. diferente a M. reptans (Hedw.) Cardot, la especie más frecuente del género en los bosques montanos de las yungas de Argentina (NOA). Este ejemplar ha sido identificado como Mittenothamnium reduncum (Schimp. ex Mitt.) Ochyra, una especie neotropical que se conocía previamente desde México hasta Bolivia y Brasil (Churchill \& Linares, 1995; Hedenäs, 2003).

En esta contribución se registra por primera vez a Mittenothamnium reduncum para la Argentina, en la provincia de Tucumán, la cual se describe, ilustra, comenta brevemente y se compara con $M$. reptans (Tabla 1 ).

\section{Materiales y MÉtodos}

Los especímenes estudiados provienen de colecciones propias realizadas en el Parque Nacional Campo de Los Alisos depositados en el herbario LIL. Las mismas fueron comparadas con material depositado en herbarios nacionales e internacionales (JE, LIL y MO) de M. reptans de la misma localidad, y con ejemplares de $M$. reduncum de otras localidades. Las muestras fueron estudiadas mediante técnicas convencionales para briófitas (Frahm, 2003). 


\section{Tabla 1. Diferencias morfológicas entre las 2 especies de Mittenothamnium encontradas en el Parque Nacional Campo de los Alisos.}

\begin{tabular}{|c|c|c|}
\hline & Mittenothamnium reduncum & Mittenothamnium reptans \\
\hline Tallos. & $\begin{array}{c}\text { Tallos simples, decumbentes } \\
\text { o subascendentes. }\end{array}$ & Tallos estipitados, arqueados. \\
\hline $\begin{array}{l}\text { Disposicion de las } \\
\text { hojas en las ramas. }\end{array}$ & Ramas densamente foliadas. & Ramas laxamente foliadas. \\
\hline Hojas de los tallos. & $\begin{array}{c}\text { Falcado secundas, fuertemente } \\
\text { cóncavas; margen entero } \\
\text { o denticulado apicalmente; } \\
\text { nunca decurrentes. }\end{array}$ & $\begin{array}{l}\text { Erectas, ampliamente extendidas; } \\
\text { margen serrulado a serrado, rara vez } \\
\text { entero; en ocasiones decurrentes. }\end{array}$ \\
\hline Hojas de las ramas. & $\begin{array}{c}\text { Ovado u oblongo lanceoladas } \\
\text { a veces triangulares, corto } \\
\text { acuminadas, algunas asimétricas. }\end{array}$ & $\begin{array}{l}\text { Ovado lanceoladas, acuminadas, } \\
\text { generalmete simétricas. }\end{array}$ \\
\hline
\end{tabular}

\section{Resultados}

\section{Tratamiento taxonómico}

Mittenothamnium reduncum (Schimp. ex Mitt.) Ochyra, Fragm. Florist. Geobot. 44: 256. $1999 \equiv$ Ctenidium reduncum Schimp. ex Mitt., J. Linn. Soc. Bot. 12: 509. 1869. 三 Hygrohypnum reduncum (Schimp. ex Mitt.) N. Nishim., J. Hattori Bot. Lab. 58: 73. 1985. Fig. 1.

Plantas medianas en tapices densos, oscuras en la base, pardo verdosas a amarillentas, a veces doradas hacia los ápices. Tallos no estipitados, irregularmente ramificados, 1,5-3,0 cm de largo; en sección transversal con esclerodermis marcada de 3-4 filas de células rodeando células grandes de paredes delgadas, sin cordón central; rizoides pardos oscuros, lisos, irregularmente ramificados; pseudoparafilos foliosos ancho-triangulares; parafilos ausentes. Hojas notoriamente falcado secundas, a veces flexuosas en el ápice al estado seco, erecto-patentes al estado húmedo, ovado u oblongo lanceoladas o triangulares, cóncavas, algunas asimétricas; ápice agudo, corto acuminado a veces curvado; hojas del tallo de 0,7-1,1 x 0,3$0,5 \mathrm{~mm}$, más grandes que las de las ramas $(0,5-0,8$ x $0,3 \mathrm{~mm}$ ); margen plano a incurvado, entero a denticulado hacia el ápice; costa doble, corta, ocupando 1/3 (-1/2) de la longitud de la hoja o menos, a veces ausente; células de la lámina flexuosas, romboidales, a veces oblongas hacia los márgenes, paredes ligeramente engrosadas, lisas (prorulosas solamente las células apicales de las hojas de las ramas), células del ápice de 18,0-34,0x 5,0-9,0 $\mu \mathrm{m}$, células de la lámina media de 27,5-40,0 (- 57,5) x 4,0- 6,25 (- 7,5) $\mu \mathrm{m}$, células basales de 25,0-62,5 x 3,75-7,5 $\mu \mathrm{m}$; células alares corto rectangulares a subcuadradas en grupos reducidos. Esporofito no observado (ver descripción de Hedenäs, 2003).

Ecología y distribución. Esta especie ha sido encontrada en el bosque montano de las yungas, habita en sitios cercanos a cursos de agua, sobre rocas. Tiene distribución neotropical, se encuentra en México, Honduras, Panamá, Costa Rica, Colombia, Venezuela, Ecuador, Perú, Bolivia, Brasil y alcanza su límite más austral en el noroeste de Argentina.

Comentarios. Mittenothamnium reptans es una especie común del género en el bosque montano, asimismo en el Parque Nacional Campo de Los Alisos donde es abundante y se encuentra como epífito, en raíces expuestas y sobre troncos, mientras que $M$. reduncum aparece restringido a áreas cercanas a cursos de agua sobre rocas, asociado a Orthostichella versicolor (Müll. Hal.) B. H. Allen \& W. R. Buck.

Material examinado. Mittenothamnium reduncum. ARGENTINA. Tucumán, Depto. Chicligasta, Parque Nacional Campo de Los Alisos, 


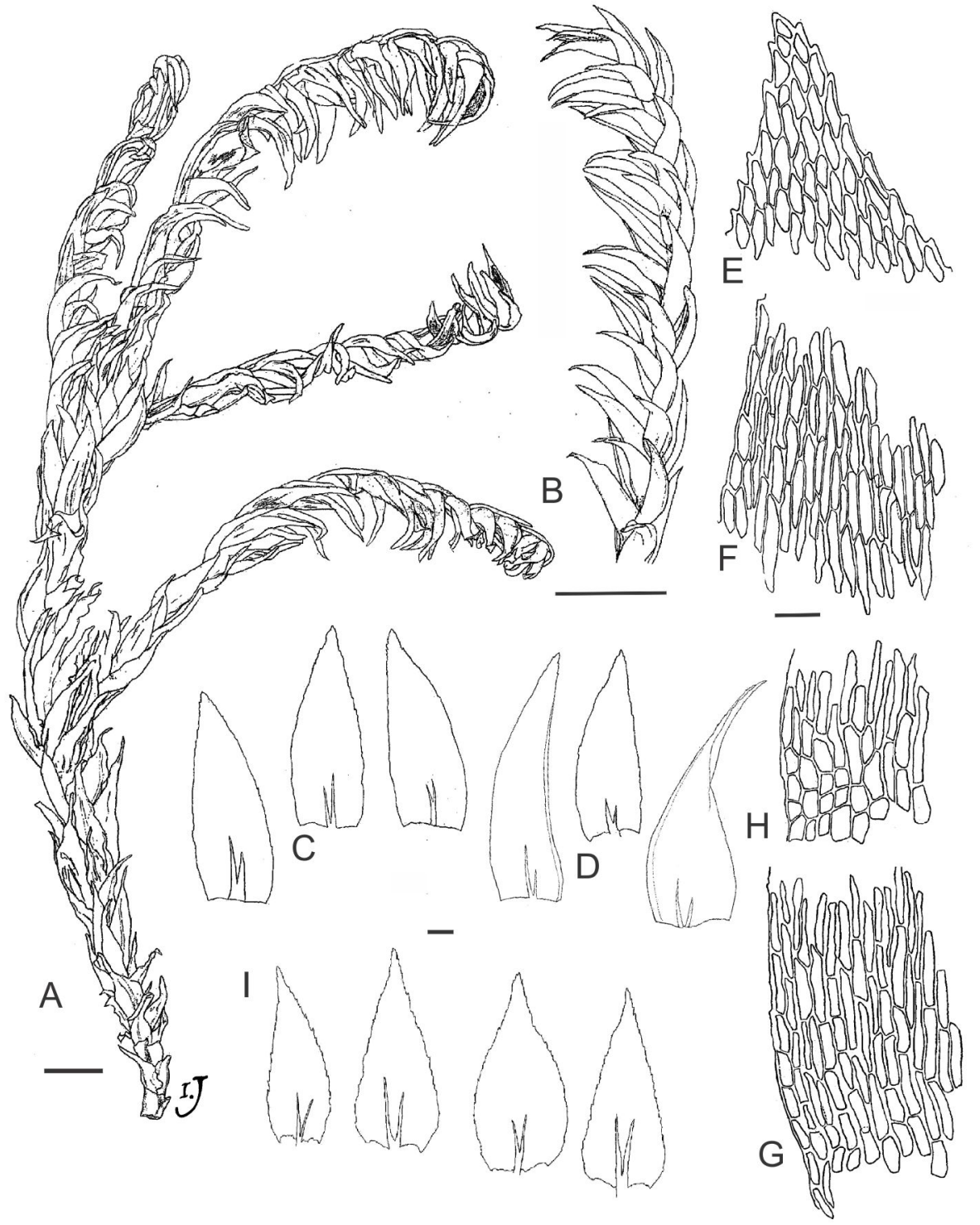

Fig. 1. Mittenothamnium reduncum (Schimp. ex Mitt.) Ochyra. A, aspecto de la planta. B, detalle de una rama al estado húmedo. $\mathrm{C}$, hojas de las ramas. $\mathrm{D}$, hojas del tallo. $\mathrm{E}$, células del ápice foliar. $\mathrm{F}$, células medias de la hoja. $G$, células basales de la hoja. $\mathrm{H}$, detalle de las células alares. Escala: $\mathrm{A}-\mathrm{B}=0,5 \mathrm{~mm} ; \mathrm{C}-\mathrm{H}=$ $1 \mu \mathrm{m}$. De G. Suárez 1635 (LIL). Mittenothamnium reptans (Hedw.) Cardot I, hojas de las ramas. Escala I= $1 \mu \mathrm{m}$. De G. Suárez 1676 (LIL).

arroyo Las Cañas, $27^{\circ} 17^{\prime} 13,9^{\prime \prime} \mathrm{S} ; 65^{\circ} 52^{\prime} 43,3^{\prime \prime} \mathrm{W}$, $904 \mathrm{~m}$, en talud y suelo, 02/11/2012, G. Suárez 1635 (LIL), 1637 (LIL). Depto. Chicligasta, rain forest along Rio Cochuña between Concepcion and Cochuña, on shady rock, ca. $1000 \mathrm{~m}$ elev., 5 january 1989, Schäfer-Verwimp \& Verwimp 10771 (JE, as Hygrohypnum reduncum). BOLIVIA.
Chuquisaca, Belisario Boeto. Nuevo Mundo; ca. $24 \mathrm{~km}$ Villa Serrano Nuevo Mundo. Bosque Montano de Tucumano-Boliviano (Podocarpus, Siphoneugena, Cinnamomum), $19^{\circ} 00^{\prime} 20^{\prime}$ S 64¹9'54" W, 2450-2500 m, musgo sobre raíz de árbol, 13/05/2004, S. Churchill et al. 22909 (MO, LIL 55369). 
Material examinado. Mittenothamniun reptans. ARGENTINA. Tucumán, Depto. Chicligasta, Parque Nacional Campo de Los Alisos, Puesto Santa Rosa, $27^{\circ} 17^{\prime} 13,9^{\prime \prime}$ S; 65 52' 43,3” W, 904 m, epífito, 02/11/2012, G. Suárez 1618 (LIL); en raíz expuesta, G. Suárez 1655 (LIL); Puesto Los Chorizos, $27^{\circ} 15^{\prime}, 30,8^{\prime \prime} \mathrm{S}$; 65 $65^{\circ}$ 53 32,5” W, $1114 \mathrm{~m}$, sobre tronco de Mirtaceae, 02/11/2012, G. Suárez 1676 (LIL). "Microthamnium tapes, Cuesta de Sn Rosa, Flora von Argentinien, 18 Juni 1873, P. G. Lorentz s.n.” (JE).

\section{Agradecimientos}

Los autores desean agradecer a la Administración de Parques Nacionales, al Dr. W. Buck por la confirmación de la especie y a la Lic. Inés Jaume (Sección Iconografía, FML) por la realización de la lámina. Este trabajo fue financiado por los proyectos PIUNT y PICT 0810.

\section{Bibliografia}

CHURCHILL, S. P. \& C. E. LINARES. 1995. Prodromus Bryologiae Novo-Granatensis. Biblioteca "José Gerónimo Triana" 12: 1-924.

COLOTTI, M. T. \& G. M. SUAREZ. 2017. Novedades sobre la familia Ditrichaceae (Bryophyta) en los pisos superiores de las yungas subtropicales de Argentina. Bol. Soc. Argent. Bot. 52: 277- 289.

FRAHM, J. P. 2003. Manual of tropical bryology. Trop. Bryol. 23: 1-200.

HEDENÄS, L. 2003. Amblystegiaceae (Musci). Flor. Neotrop. Monograph 89: 1-108.

Recibido el 30 de agosto de 2017, aceptado el 27 de octubre de 2017. Editor: Guillermo Suárez. 